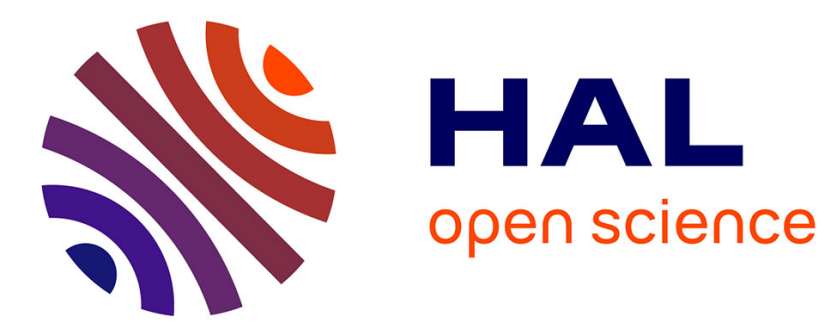

\title{
ESR in the two phases of the magnetic semiconductor GaCr4S 8
}

\author{
P. Huguet, Georges Alquié, M. Potel, M. Sergent
}

\section{To cite this version:}

P. Huguet, Georges Alquié, M. Potel, M. Sergent. ESR in the two phases of the magnetic semiconductor GaCr4S 8. Journal de Physique Lettres, 1983, 44 (10), pp.393-401. 10.1051/jphyslet:019830044010039300 . jpa-00232208

\section{HAL Id: jpa-00232208 https://hal.science/jpa-00232208}

Submitted on 1 Jan 1983

HAL is a multi-disciplinary open access archive for the deposit and dissemination of scientific research documents, whether they are published or not. The documents may come from teaching and research institutions in France or abroad, or from public or private research centers.
L'archive ouverte pluridisciplinaire $\mathbf{H A L}$, est destinée au dépôt et à la diffusion de documents scientifiques de niveau recherche, publiés ou non, émanant des établissements d'enseignement et de recherche français ou étrangers, des laboratoires publics ou privés. 
Classification

Physics Abstracts

$76.30-76.50$

\title{
ESR in the two phases of the magnetic semiconductor $\mathbf{G a C r}_{4} \mathbf{S}_{8}$
}

\author{
P. Huguet, G. Alquié \\ Laboratoire de dispositifs Infrarouge et de Résonance Ivagnétique (*), \\ Université Pierre et Marie Curie, Tour 12, 2e étage, 4, place Jussieu, 75230 Paris Cedex 05, France
}

\author{
M. Potel and M. Sergent \\ Laboratoire de Chimie Minérale B, L. A. 254, Campus de Beaulieu, Avenue du Général Leclerc, \\ 35042 Rennes Cedex, France
}

(Reçule 29 décembre 1982, révisé le 10 mars 1983, acceptéle 24 mars 1983)

\begin{abstract}
Résumé. - Des résultats nouveaux sur les deux phases cristallographiques du semiconducteur magnétique $\mathrm{GaCr}_{4} \mathrm{~S}_{8}$ sont présentés; ils ont été obtenus par résonance de spins électroniques dans les états paramagnétique et magnétique ordonné de ces composés. Un comportement typique des transitions de phases magnétiques est observé; il est caractérisé par un déplacement et un élargissement du signal de résonance, ainsi que par une modification de sa forme. Ces effets sont interprétés en relation avec la présence de fluctuations d'aimantation.
\end{abstract}

\begin{abstract}
We present new results obtained from ESR experiments in the paramagnetic and magnetic ordered states of both crystallographic phases of the magnetic semiconductor $\mathrm{GaCr}_{4} \mathrm{~S}_{8}$. We observe a critical behaviour typical of magnetic phase transitions, resulting in a shift and a broadening of the resonance line as well as a distortion of its shape. These features are discussed and related to the presence of magnetization fluctuations.
\end{abstract}

\section{Introduction.}

Chalchogenide spinels of type $\mathrm{MCr}_{2} \mathrm{X}_{4}$ such as $\mathrm{CdCr}_{2} \mathrm{Se}_{4}$ or $\mathrm{HgCr}_{2} \mathrm{Se}_{4}$ have been extensively studied because they exhibit both magnetic and semiconducting properties, with a strong coupling between these two features [1].

$\mathrm{GaCr}_{4} \mathrm{~S}_{8}$ is a cation deficient spinel where similar properties can be expected. This compound has the atomic arrangement $\mathrm{Ga}_{2 / 3} \square_{1 / 3} \mathrm{Cr}_{2} \mathrm{~S}_{4}$ and presents two phases. Both exhibit semiconducting properties. The high temperature annealed phase $(\beta)$ has a vacancy disordered structure and has been found ferromagnetic with a Curie temperature about $20 \mathrm{~K}$. The low temperature phase $(\alpha)$ has a vacancy ordered structure and seems antiferromagnetic with a Néel point about $10 \mathrm{~K}[2,4]$. Nevertheless, no divergence is observed in the susceptibility curves, and in the absence of thermodynamical measurements, the critical points are not well defined. Crystallographic

(*) Equipe associée au C.N.R.S. $\mathrm{n}^{\circ} 365$. 
structure and static magnetic properties have been studied in references [2] and [4]. We present in this paper our dynamic measurements through ESR experiments.

\section{Experimental techniques and structural results.}

The ESR measurements have been performed with a superheterodyne X-band (10.9 GHz) spectrometer with temperature ranging from $1.5 \mathrm{~K}$ to $300 \mathrm{~K}$, measured with suitably calibrated resistors. The $\mathrm{GaCr}_{4} \mathrm{~S}_{8}$ powder was put in a silica tube against the wall of a $\mathrm{TE}_{102}$ cavity. Gyromagnetic factors were determined calibrating the resonance field against a $g$-marker (DPPH).

The elaboration of the samples and their characterization were performed at the " Laboratoire de chimie minérale $B »$. The powder samples have been prepared by firing stoichiometric mixtures; then a heat treatement under $\mathrm{H}_{2} \mathrm{~S}$ atmosphere was performed. The $\dot{\alpha}$-phase was obtained by annealing the samples at $850^{\circ} \mathrm{C}$ and the $\beta$-phase by annealing the $\alpha$-phase at $1180{ }^{\circ} \mathrm{C}$.

We will now summarize some crystallographic properties. The X-ray pattern of the $\alpha$-phase $\left(a_{\text {cubic }}=9.90 \mathrm{~A}\right)$ is similar to that of $\mathrm{Mo}_{4}$ tetraheural clusters compounds [3]. The $\beta$-phase $\left(a_{\text {cubic }}=9.88 \AA\right)$ exhibits a pattern characteristic of a f.c.c. lattice with, in this case, an extinction of the $(h k 0)(0 k l),(h 0 l)$ spots for $h+k=4 n+2$.

The $\alpha$ and $\beta$ phases structure is isotype to the $\mathrm{AB}_{2} \mathrm{X}_{4}$ spinel structure which belongs to the space group $\mathrm{Fd} 3 \mathrm{~m}$. It exhibits $\mathrm{Cr}_{4} \mathrm{~S}_{4}$ cubes similar to the $\mathrm{B}_{4} \mathrm{X}_{4}$ ones and $\mathrm{Ga}_{x} \mathrm{~S}_{4}$ tetrahedrons similar to the $\mathrm{AX}_{4}$ tetrahedrons. Nevertheless :

- the position $(32 c)$ of the spinel X-anions is split into two positions $(16 e)(x, x, x)$,

- the position $(8 a)$ of the spinel A-atoms is split into two positions with a multiplicity of 4 : one $(4 a)$ at $(0,0,0)$, the other $(4 c)$ at $(3 / 4,3 / 4,3 / 4)$,

- the position of the spinel B-atoms becomes $(16 e)$ at $(x, x, x)$, leading to two types of $\mathrm{Cr}-\mathrm{Cr}$ spacings and to two basis $\mathrm{Cr}_{4} \mathrm{~S}_{4}$.

A more precise study of the structure of the $\alpha$-phase indicates that a $\mathrm{Ga}$ atom is located at $4 a(0,0,0)$ while the Ga occupancy is only $1 / 3$ at $4 c(3 / 4,3 / 4,3 / 4)$. So the formula of this phase can be written $\mathrm{Ga}_{0.33} \mathrm{GaCr}_{4} \mathrm{~S}_{8}$.

The $\beta$-phase is characterized by an equal Ga occupancy on the two sites, which corresponds to the formula $\mathrm{Ga}_{0.66} \mathrm{Ga}_{0.66} \mathrm{Cr}_{4} \mathrm{~S}_{8}$. Nevertheless as shown from structural resolutions, the spatial group remains $\mathrm{F} \overline{4} 3 \mathrm{~m}$ for the two phases with two types of $\mathrm{Cr}-\mathrm{Cr}$ spacings $(3.35 \AA$ inside the $\mathrm{Cr}_{4} \mathrm{~S}_{4}$ basis and $3.64 \AA$ for the $\mathrm{Cr}_{4} \mathrm{~S}_{4}$ basis which link them) [4].

\section{Experimental results and analysis.}

The main features shown by the ESR lines of both phases are the following :

1) The line shapes are Lorentzian from $300 \mathrm{~K}$ to $40 \mathrm{~K}$ and become asymmetric near the ordering temperature(Fig. 1). It has not been possible to work out quantitative results below a temperature of about $30 \mathrm{~K}$, since the lines are too large and shifted to very low fields.

2) A pronounced broadening of the linewidth $\Delta H_{\mathrm{pp}}$ is observed when approaching the ordered state from the paramagnetic side (Fig. 2).

3) The resonance field is constant above $40 \mathrm{~K}$ and shows a rapid decrease below (Fig. 3).

Some differences are observed between the two phases :

1) The high temperature linewidth of the $\beta$-phase is narrower compared to that of the $\alpha$, and exhibits a decrease between $300 \mathrm{~K}$ and $110 \mathrm{~K}$. On the opposite the $\alpha$-phase linewidth remains constant between 200 and $300 \mathrm{~K}$, but shows an almost linear decrease between 200 and $50 \mathrm{~K}$.

2) Though a similar critical behaviour for both compounds is observed, magnetic order effects seem to appear at lower temperatures for the $\alpha$-compound compared to the $\beta$ (Figs. 2,3 ). 


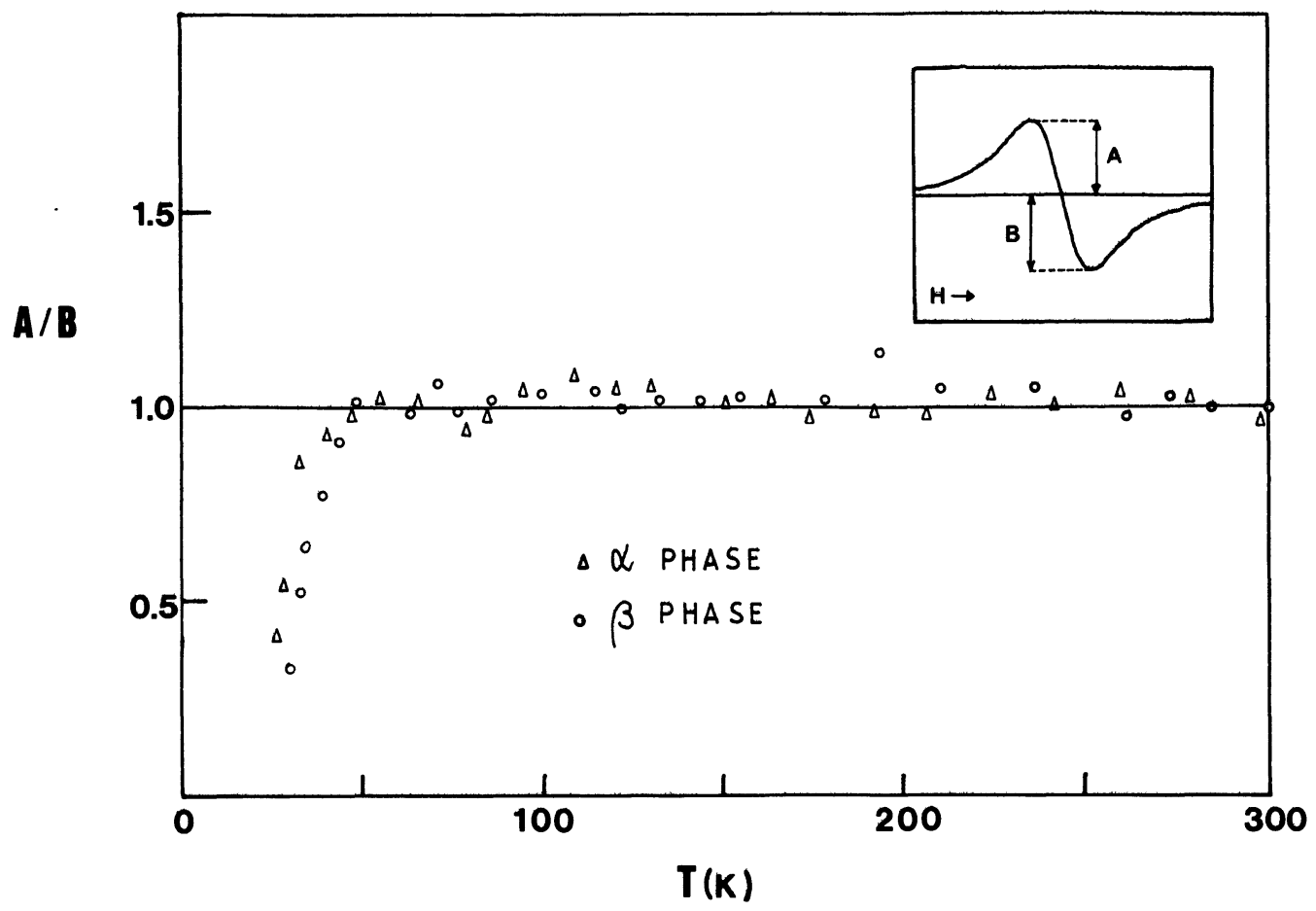

Fig. 1. - Temperature dependence of the asymmetry ratio $A / B$ for both phases. Insert : definition of parameters $A$ and $B$.

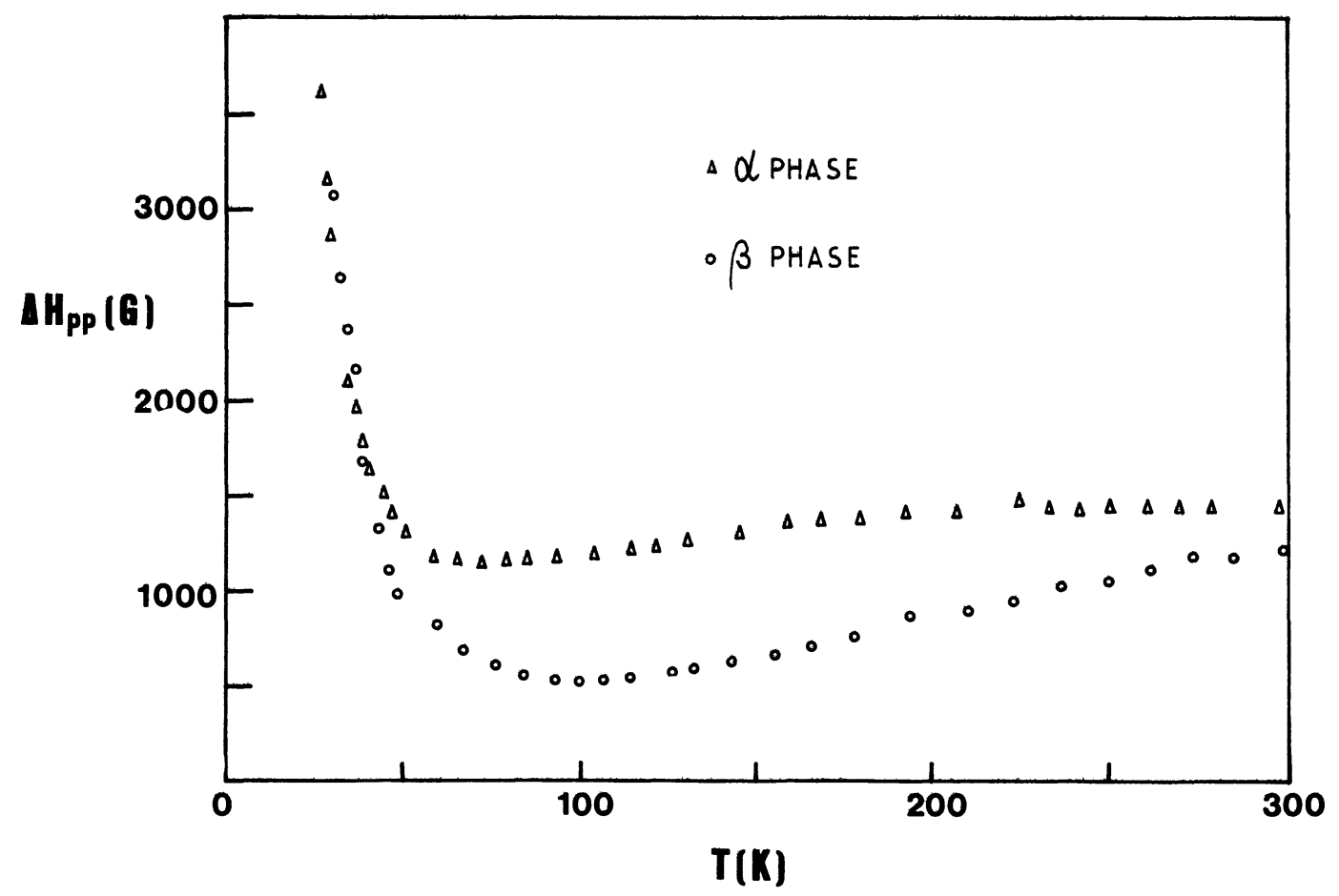

Fig. 2. - Temperature dependence of the linewidth $\Delta H_{\mathrm{pp}}$ for both phases. 


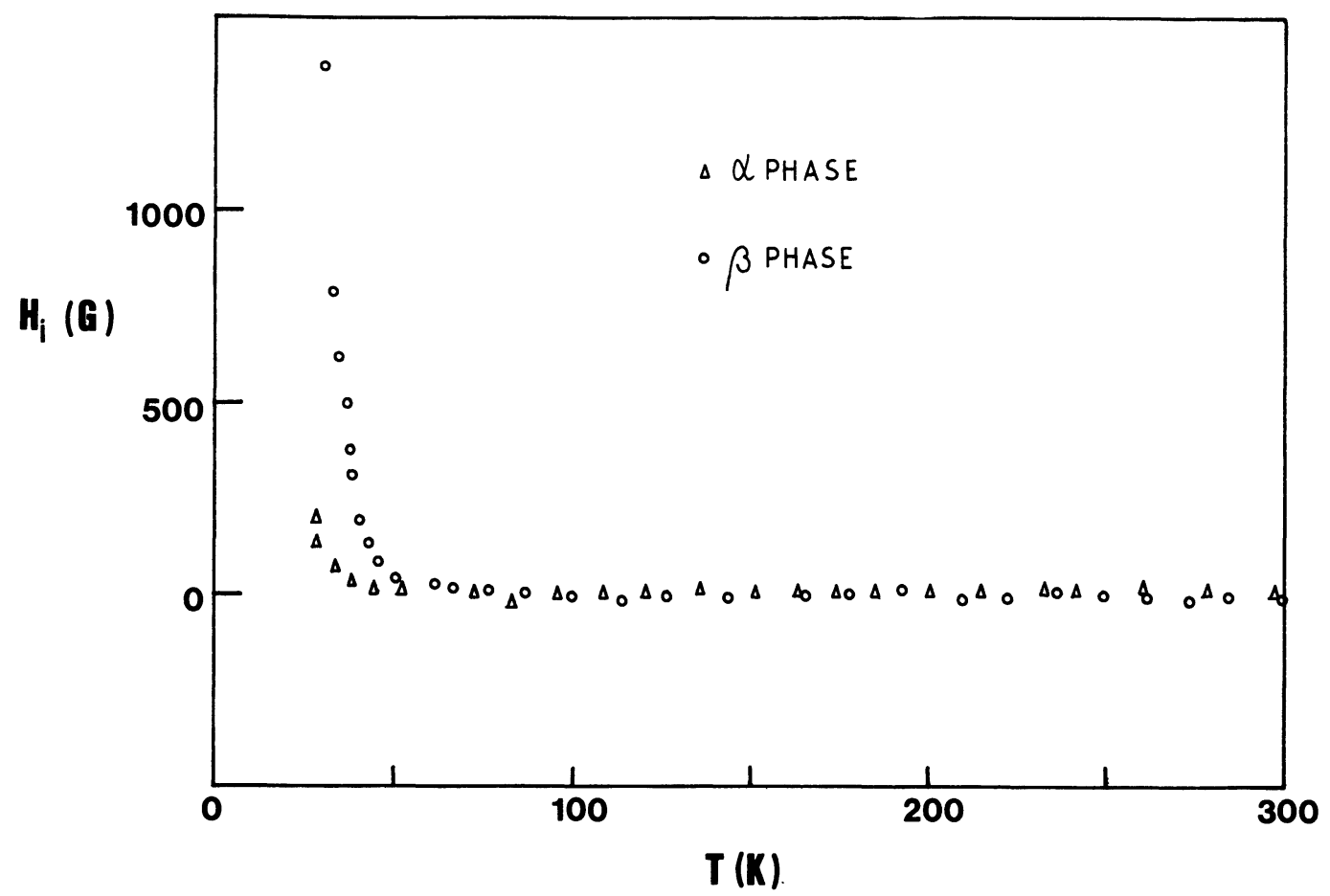

Fig. 3. - Temperature dependence of the internal field $H_{\mathrm{i}}$ for both phases.

The temperature behaviour of the linewidth in the various regimes are fitted using a leastsquares fit method. In the paramagnetic regime $(T>100 \mathrm{~K})$ the linewidth of both compounds are well described by an exponential dependence with temperature :

$$
\Delta H_{\mathrm{pp}}=\Delta H_{0}+\delta H_{\infty} \exp (-\theta / T) .
$$

The coefficients as well as the average values of the $g$-factor in this high temperature region are listed in table $I$.

Near the magnetic ordering temperature, a Huber's law is used to fit the thermal linewidth dependences :

$$
\Delta H_{\mathrm{pp}}=\Delta H_{\infty}+A\left(\frac{T_{\mathrm{c} \Delta H}}{T-T_{\mathrm{c} \Delta H}}\right)^{\alpha_{\Delta H}} .
$$

The various parameters we obtained are listed in table II. In this temperature range, the resonance lines no longer present a Lorentzian shape; the resonance field $H_{\mathrm{R}}$ is assumed to be given by the

Table I. - Parameters of the high temperature ESR tines.

\begin{tabular}{|c|c|c|c|c|}
\hline & $\Delta H_{0}$ & $\delta H_{\infty}$ & $\theta$ & $g$ (high temp.) \\
\hline$\alpha$ & 300 & 1350 & $40 \mathrm{~K}$ & $1.981 \pm 0.002$ \\
\hline$\beta$ & 180 & 2100 & $220 \mathrm{~K}$ & $1.973 \pm 0.002$ \\
\hline
\end{tabular}


Table II. - Parameters of the critical behaviour of the ESR line. The lack of experimental data for the internal field of the $\alpha$-phase did not allow us to extract meaningful critical coefficients.

\begin{tabular}{|c|c|c|c|c|c|c|c|c|}
\hline & $\Delta H_{\infty}$ & $A$ & $T_{\mathrm{c} \Delta H}$ & $\alpha_{\Delta H}$ & $H_{0}$ & $B$ & $T_{\mathrm{cH}_{i}}$ & $\alpha_{H_{i}}$ \\
\hline$\alpha$ & 370 & 1226 & 20.9 & 0.7 & 3900 & - & - & - \\
\hline$\beta$ & 264 & 1286 & 19.9 & 1.3 & 3910 & 8.7 & 22.4 & 2.7 \\
\hline
\end{tabular}

mean value of the two peaks fields of the signal. The observed shift of the resonance field, compared to the high temperature value $H_{0}$, is governed by an internal field $H_{\mathrm{i}}$ that is built up when approaching the transition temperature [5] :

$$
H_{\mathrm{i}}=H_{0}-H_{\mathrm{R}} .
$$

Its thermal behaviour is found to be described by a Huber's law

$$
H_{\mathrm{i}}=B\left(\frac{T_{\mathrm{cH}_{\mathrm{i}}}}{T-T_{\mathrm{cH}}}\right)^{\alpha_{\mathrm{H}_{\mathrm{i}}}} .
$$

The respective coefficients are given in table II.

\section{Discussion.}

4.1 High TEMPERATURE. - In the high temperature region, the line is broad and Lorentzian for both compounds. Due to dipolar interactions, the line should be Gaussian, with a peak-topeak width given for a powder by :

$$
\left(\Delta H_{\mathrm{pp}}\right)_{\mathrm{dip} .}=2 \sqrt{M_{2}}=2 \sqrt{3 / 5} g \mu_{\mathrm{B}} \sqrt{S(S+1) \sum_{j}\left(r_{j k}\right)^{-6}} .
$$

For both phases, we obtain approximatively $\Delta H_{\mathrm{pp}} \simeq 3200 \mathrm{G}$. It is then obvious that the line is exchange-narrowed (Lorentzian, $600<\Delta H<1000 \mathrm{G}$ ). The strength of the exchange interaction responsible of such a narrowing can be evaluated using the Anderson's result :

$$
\left(\Delta H_{\mathrm{pp}}\right)_{\mathrm{dip}+\text { exch }}=(1 / 2 \sqrt{3})\left(\Delta H_{\mathrm{pp}}\right)_{\mathrm{dip}}^{2} /(J / \gamma \hbar) .
$$

Since $(J / \gamma \hbar) \gg H_{0}$, we take into account the «10/3 effect », and finally obtain, for the lowest value measured of $\Delta H_{\mathrm{pp}}(530 \mathrm{G}$ for the $\alpha$-phase) $J / k \simeq 2.5 \mathrm{~K}$. In order to compare this value with the molecular field prediction, we calculate the strength of the exchange interaction obtained from the Curie-Weiss asymptotic temperature, using :

$$
\theta=(2 / 3)(J / k) z S(S+1) \text {. }
$$

Taking into account only the 6 direct superexchange interactions (this point is discussed in general for chromium spinels in Ref. [6]), we deduce an effective value of $J$ for the $\beta$-phase $: J / k \simeq 3.4 \mathrm{~K}$ with $\theta \simeq 50 \mathrm{~K}$, in rough agreement with the value deduced from the exchange narrowing. Nevertheless, this value results in a linewidth about $390 \mathrm{G}$; the difference between this value and the experimental ones may result from other sources of broadening typical of powder samples :

1) The distribution of the crystallites shapes; it induces a distribution of demagnetizing fields, resulting in a broadening and shift of the line. This effect depends on the magnetization of the 
sample; we checked that the susceptibility is too low, in the high temperature region to allow such an effect.

2) The porosity; Schlöman [7] gave a semi-empirical expression of the additional linewidth :

$$
(\Delta H)_{\text {pores }}=1.5(4 \pi M) v / V
$$

where $v / V$ is the ratio of the volume of the pores to the volume of the sample, and $4 \pi M$ the total magnetization of the sample. We calculate the resulting corrections at different temperatures for $v / V=1$ and find that it never exceeds $10 \mathrm{G}$ in a $5 \mathrm{kG}$ field.

3) Anisotropy effects; the magnetocrystalline anisotropy has not been taken into account, since only operative below the critical points. In the paramagnetic region, the $g$-factor anisotropy is found negligible for the $\alpha$-compound, since the value of the $g$-factor for $\mathrm{Cr}^{3+}$ in a pure cubic field is 1.980 . For the $\beta$-phase, the measured $g$-factor slightly differs from this value $(g \simeq 1.973 \pm$ 0.002). This can be explained by the deformations of the cubic crystal field resulting from the occupancy of gallium sites. This is in agreement with the X-rays results, which indicate a lower symmetry for the $\beta$-phase. We estimate that the resulting additional broadening cannot exceed $100 \mathrm{G}$.

Another important feature of the resonance in the high temperature region is that the linewidth is temperature dependent; this effect is less important for the $\alpha$-phase compared to the $\beta$-phase. This may be attributed to a spin-lattice relaxation mecanism. The thermal expansion of the lattice cannot induce a relative change in the linewidth of the order of unity. In the same way, an indirect process via conduction electrons is improbable since their density is too low ( $\rho \simeq 5 \times 10^{5} \Omega \mathrm{cm}$ at $200 \mathrm{~K}$ for the $\beta$-phase). Since it is expected that the $\mathrm{Cr}^{3+}$ ions are weakly bound to the lattice ( $S$-state, in agreement with the measured $g$-value), the spin-lattice relaxation mechanism can result from a two-step process involving impurities. Such a mechanism has been evidenced in other chromium spinels where $\mathrm{Cr}^{2+}$ in octahedral sites and $\mathrm{Cr}^{3+}$ in tetrahedral sites are fast relaxing impurities [8]; their coupling with the $\mathrm{Cr}^{3+}$ ions involves phonons of low energy, localized on these paramagnetic impurities. A linewidth temperature dependence of the following form is predicted :

$$
\Delta H=\Delta H_{0}+\delta H_{\infty} \exp (-\theta / T) .
$$

This can explain our experimental results provided that the energy of these local phonons is as low as $\theta \simeq 40 \mathrm{~K}$. Such a value is roughly an order of magnitude lower than those observed in other chromium spinels; this may result from the Ga deficiency of the structure.

4.2 LOW TEMPERATURE. - For both compounds the linewidth as well as the resonant field rapidly change when the critical temperature is approached. As the magnetization always remains smaller than $50 \mathrm{G}$ for both phases [2], this effect cannot be related to demagnetizing fields. Therefore these features are attributed to the presence of critical fluctuations of the magnetization and have been analysed in terms of Huber's critical laws. Theoretical treatments have been performed, predicting the behaviour of the spin-spin relaxation rate for ferromagnets near the critical point $[10,11]$. In order to compare our measurements with there results, we plot $\Delta H_{\mathrm{pp}}$ as a function of the susceptibility given in reference [4], and obtain for the $B$-nhase between 90 and $45 \mathrm{~K}$ the law :

$$
\Delta H_{\mathrm{pp}} \propto \chi^{0.67}
$$

This is in agreement with both Huber and Kawasaki results, predicting a 3/4 exponent for the spin-spin relaxation rate. Nevertheless, a comparison between theory and our experiments 
presents two mains difficulties. First, this $\beta$-compound is not a real ferromagnet; it exhibits both competing ferromagnetic and antiferromagnetic interactions, which results in a metamagnetic or spin glass state below the critical temperature. Thus, as in other chromium spinels, $\chi(T)$ exhibits a large $\theta / T_{\mathrm{c}}$ value and $\sigma(T)$ shows a cusp below the critical temperature, which could result from a ferromagnetic-antiferromagnetic transition similar to that observed in $\mathrm{HgCr}_{2} \mathrm{~S}_{4}$ [6]. The second difficulty occurs from the appearance of an asymmetry in the resonance lines near the critical temperature, which introduces some uncertainty on the linewidth and the resonance field.

This asymmetry $(A / B<1)$ in the Lorentzian shape has been investigated. Considering the expression of the power absorbed by the sample, we find three different contributions able to modify the Lorentzian shape :

1) As $\Delta H_{n n}$ is large, one has to take into account both resonance lines corresponding to both circular polarizations occurring from the linearly polarized r.f. field. A computer simulation shows that the resulting correction leads to a weak asymmetry (a $A / B$ of about 0.7 is found for the most deformed line), but still too weak to explain alone the lineshape asymmetry near $T_{\mathrm{c}}$. 2) The absorbed power is proportional to the magnetization; since $\Delta H_{p p}$ is large, we cannot still assume that the magnetization is constant during each experiment. The resulting distortion has been calculated [12] and leads to an asymmetric line with $A / B>1$, which, clearly is not our case. 3) A field dependent susceptibility can also induce deformations [13]. But such an effect can only be operative below the magnetic ordering temperature. Therefore, though A.C. susceptibility measurements have not yet been performed, we shall not take this effect into account, since our measurements are made in the paramagnetic region. Hence we think that the strong asymmetry observed $(A / B$ values up to 0.4$)$ cannot result from neglected factors in the expression of the absorbed power but rather from physical effects produced by the external field in the material. Such an effect has been invoked by Tomita et al. [14] : they showed that the critical broadening is reduced by a strong anisotropy, in particular by the uniaxial anisotropy induced by the magnetic field in a cubic magnet. This results in a change of the effective $g$-factor and of the Lorentzian line profile. But this does not seem to account for our lineshape deformation since the prediction for a strong anisotropy is a Gaussian line profile. Another source of distortion is the effect of the magnetic field upon the magnetization fluctuations, which are the main source of broadening. In zero field near $T_{\mathrm{c}}$, the correlations are dominated by long wavelength fluctuations. These fluctuations are strongly reduced by the magnetic field. For the nearest-neighbours exchange interaction in a cubic lattice of parameter $a$, it follows from the random phase approximation that the correlation length at the critical temperature is given by [15] :

$$
\xi \simeq a\left(k_{\mathrm{B}} T_{\mathrm{c}} R / 3 g \mu_{\mathrm{B}} H_{0}\right)^{0.5}
$$

where $R$ is the relative magnetization $M(T) / M(0)$. From static magnetic measurements we can get approximatively for both phases $\xi \simeq 3.5 a$, in the field required for X-band resonance. This clearly shows how the magnetic field drastically reduces the long-range correlations in the critical region. The field dependence of $\Delta H_{\mathrm{pp}}$ and $H_{\mathrm{i}}$, even far from the critical point has been evidenced by Kötzler et al. $[9,16]$. More precisely, they showed that the linewidth is strongly reduced by the presence of the applied field. This feature does not affect the Lorentzian shape since the linewidth is small compared to the resonance field. The situation is clearly different with $\mathrm{GaCr}_{4} \mathrm{~S}_{8}$, where the line is broad; thus, during each experiment, a continuous decay of the long-range fluctuations is expected, resulting in an asymmetric line profile. The calculation of such a profile requires the knowledge of the distribution of the fluctuations, their relaxation rates and their dependence upon the magnetic field. These parameters being unknown, we have tried to calculate lineshapes using a phenomenological approach. Thus we assume that the removal of the long-range fluctuations results in a field-dependent contribution $f(H)$ to the inhomogeneous 


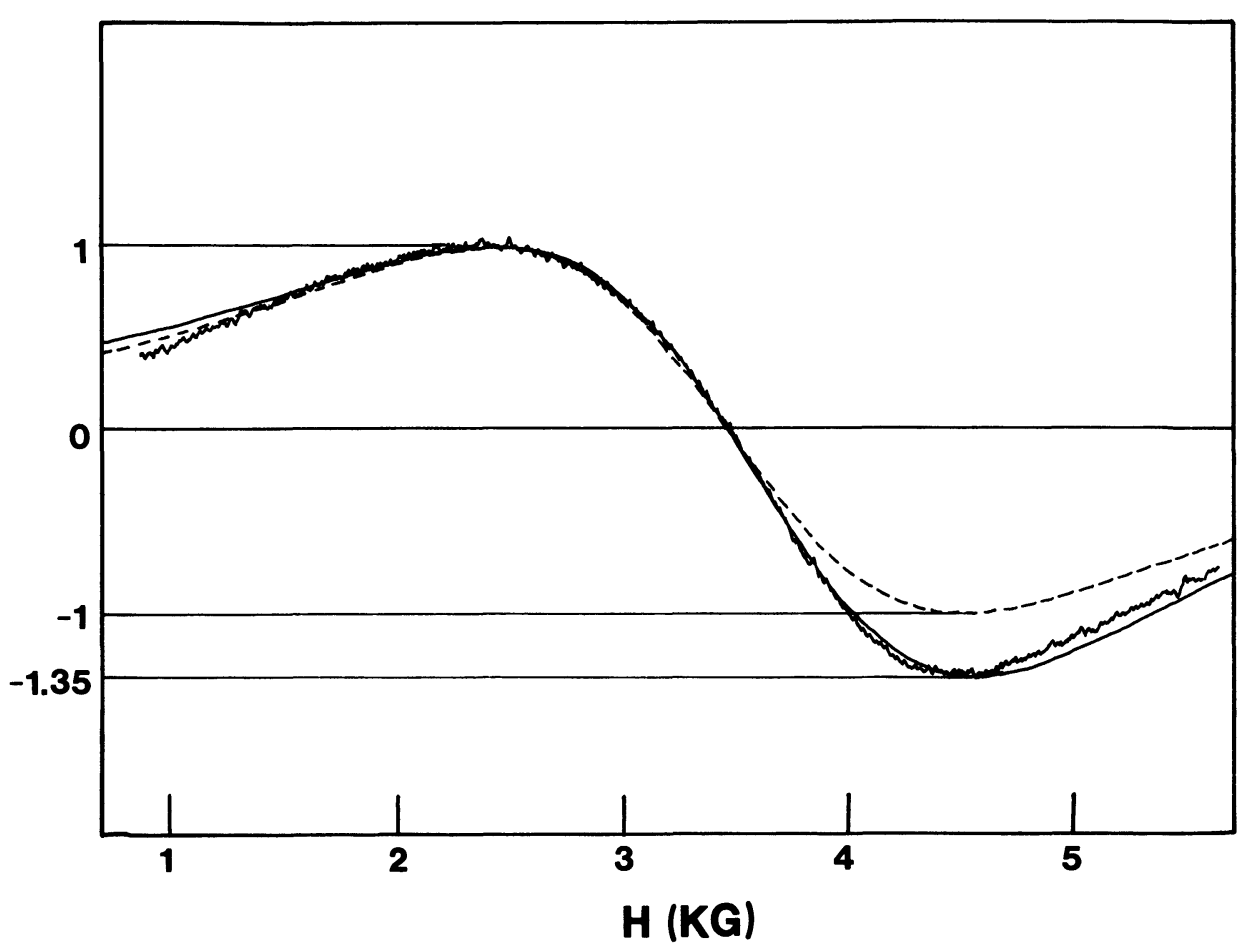

Fig. 4. - Experimental lineshape at $36 \mathrm{~K}$ for the $\beta$-phase. The asymmetry ratio is 0.74 . Dotted line is a simulation of a purely Lorentzian line. Full line is a simulation of a modified Lorentzian line, including the field-dependent linewidth due to the decay of the long-range correlations; for this fit, we used $\Delta H(H)=2682 \mathrm{G}-0.15 H$. The contribution of the $(-\omega)$ line has been neglected.

broadening of the line, sothat the lineshape can be described by a Lorentzian profile modified by a field dependence on the linewidth such as :

$$
\Delta H(H)=\Delta H_{0}-f(H) .
$$

We find a good agreement between experimental lines with $A / B>0.7$ and the calculated shapes using a linear function $f(H)$. In figure 4 is shown such a simulation and the experimental line. As the temperature decreases, the coefficients of $\Delta H(H)$ increase; this results from the increase of the long-range correlations when approaching $T_{\mathrm{c}}$. For strongly deformed lines $(A / B<0.7)$, a linear correction in $\Delta H$ no more fits the experimental line; one has to take more rapidly increasing functions, and cannot still assume that the $(-\omega)$ line is negligible.

This phenomenological approach should enable us to extract the desired zero-field limit for $\Delta H_{\mathrm{pp}}$ and $H_{\mathrm{i}}$, but in the absence of the right analytical form of $f(H)$, their determination is somewhat uncertain. Following Kötzler et al., we made ESR measurements at a lower frequency $(1 \mathrm{GHz})$, in order to weaken the influence of the magnetic field. We have found unworkable broad and deformed lines. In the impossibility of knowing the true parameters of the lines near $T_{\mathrm{c}}$, we used the rough experimental results to extract critical laws.

For the ferromagnetic $\beta$-phase, the values of $T_{\mathrm{c}}$ obtained this way, through the linewidth and through the internal field (Table II), are in a reasonable agreement with the critical temperatures given by the static measurements. On the opposite, the values we obtained for the antiferromagnetic $\alpha$-phase are significantly larger than the value deduced from the static measurements. This can result from the influence of the static magnetic field on the internal energy. Thermody- 
namical measurements are then required to conclude. A good agreement is also obtained between the temperature where critical phenomena begin to appear in the ESR linewidth and that of the departure from the Curie-Weiss law of the static measurements (about $4 T_{\mathrm{c}}$ for the $\beta$-phase and $5 T_{\mathrm{N}}$ for the $\alpha$-phase) ; these temperatures are interpreted as the points of appearance of shortrange correlations. On the other hand, the critical behaviour of the internal field begins at much lower temperatures. This seems to indicate that a minimum size is required for the magnetic clusters to shift the resonance field. In fact, the shift arises at temperatures close to those of the appearance of asymmetric lines. This is in agreement with our hypothesis that these asymmetric lines result from the effect of the magnetic field on the long-range correlations.

4.3 Conclusion. - As a conclusion, we can say that the high temperature behaviour of the line can be explained by a cross-relaxation mechanism similar to the one observed in other chromium spinels. At lower temperatures, the presence of short-range fluctuations far from the critical points, perceived through the static measurements, are confirmed, as well as the existence of long-range correlations close to the critical points. A qualitative interpretation of the appearance of deformed lines has been given, pointing out the effect of the magnetic field on these long-range fluctuations.

\section{References}

[1] Gurevich, A. G., Yakoulev, Yu. M. et al., Sov. Phys. Semicond. 9 (1975) 1-5.

[2] NaKatini, I., J. Solid State Chem. 35 (1980) 50-58.

[3] Perrin, C., Chevrel, R., Sergent, M., C.R. Hebd. Sean. Acad. Sci. 280 C (1975) 949-52.

[4] Ben YaICH, H., thesis (1981), Université de Rennes I.

Ben Yaich, H., Jegaden, J. C., Potel, M., Sergent, M., to be published in Mat. Res. Bull.

[5] Oseroff, S. B., Phys. Rev. B 25 (1982) 6584-94.

[6] Baltzer, P. K., Robbins, P. J., Lopatin, E., Phys. Rev. 151 (1966) 367-377.

[7] SchlömanN, E., Conf. on Mag. and Mag. Mat. AIEE Spc. Pub. T-91 (1956) 600.

[8] Stasz, J., Acta Phys. Pol. A 51 (1977) 525-531.

[9] Kötzler, J., Philipsborn, H. von, Phys. Rev. Lett. 12 (1978) 790-792.

[10] KaWASAKI, K., in Phase Transition \& Critical Phenomena edited by C. Domb \& Green, vol. 5A (Academic Press, New-York) 1976.

[11] Huber, D. L., J. Phys. Chem. Solids 32 (1971) 2145-2149. Phys. Rev. B 6 (1972) 3180-3189.

[12] Hedgcock, F. T., Raudorf, T. W., Can. J. Phys. 50 (1972) 579-582.

[13] Vaknin, D., Davidov, D., Nieuwenhuys, G. J., Hoekstra, F. R., Barberis, G. E., Mydosh, J. A., Physica 108B (1981) 765-766.

[14] Tomita, T., Kawasaki, K., Prog. Theor. Phys. Rev. 44 (1970) 1173-1191.

[15] Leichner, P. K., Richards, P. M., Phys. Rev. B 7 (1973) 453-457.

[16] Kötzler, J., Scheitte, J., Phys. Lett. 72A (1979) 473-475. 